\title{
Evaluación a profesores en las competencias matemáticas de Educación Media, Juigalpa, 2014
}

\author{
Tonys Romero Díaz ${ }^{1}$ \\ José Eligio Guzmán Contreras ${ }^{2}$
}

\section{Resumen}

Este artículo fue producto de un diagnóstico, obtenido al aplicar una prueba de matemática, que consistió en el examen de admisión para el nuevo ingreso 2014 que aplica la Universidad Nacional Autónoma de Nicaragua, Managua. Se utilizó una muestra de veinte profesores del municipio de Juigalpa, Chontales, quienes de manera voluntaria aplicaron al examen. Se realizó un análisis de discriminación a cada uno de los ítems que componían la prueba, así como a todo el examen. Paralelamente al análisis cuantitativo, se identificó una serie de problemas en la resolución del examen a través de un análisis cualitativo de cada uno de los ejercicios planteados. Los resultados reflejaron que los profesores de secundaria del municipio de Juigalpa presentan dificultades en la resolución de ejercicios que son evaluados en los exámenes de admisión de la UNAN-Managua. Las áreas de estudio más afectadas en el examen fueron: la geometría euclidiana, los logaritmos, la teoría de conjuntos, la estadística, funciones reales y la trigonometría. Se recomienda establecer una estrategia de trabajo en conjunto UNAN-Managua y el Ministerio de Educación que refuerce la competencia de los docentes en pro de beneficiar al estudiantado de secundaria para los futuros exámenes de admisión.

Palabras clave: Examen admisión; índices de discriminación; competencia docente; MINED.

\section{Summary}

This article was the result of a diagnostic that was obtained by applying a math test, which consisted of the admission test for new students of the year 2014 that the National Autonomous University of Nicaragua (Managua) applies. We used a sample of twenty teachers from the municipality of Juigalpa, Chontales who voluntarily applied the test. The discrimination analysis was done to each of the items that made up the test, as well as the entire test. Parallel to the quantitative analysis, a series of problems were identified in resolving the test through a qualitative analysis of each of the exercises. The results showed that secondary teachers in the municipality

1 Doctor en Educación e Intervención Social. Profesor de Matemática de la Universidad Nacional Autónoma de Nicaragua, Managua. Departamento de Ciencias de Educación y Humanidades. UNAN-Managua, FAREM Chontales. Correo: tonyromerong@hotmail. com

2 Master en Formación de Formadores de Maestros de Educación Básica y Media. Profesor de Matemáticas de la Universidad Nacional Autónoma de Nicaragua, Managua. Departamento de Ciencias de Educación y Humanidades. UNAN-Managua, FAREM Chontales. Correo: eligioinach@hotmail.com 
of Juigalpa presents difficulties in resolving the exercises that are evaluated in the admission test of the UNAN-Managua. The study areas that are most affected in the test were: Euclidean geometry, logarithms, set theory, statistics, real functions and trigonometry. Therefore, based on the results, it is recommended to establish a strategy of working together UNAN-Managua and the Ministry of Education to strengthen the teacher's competence in order to benefit secondary students for future admissions tests.

Keywords: Admission test; levels of discrimination; teaching competence; MINED.

\section{Introducción}

Los educadores viven en una presión casi implacable por demostrar su eficacia. Desafortunadamente, el principal indicador con el cual la mayoría de las comunidades evalúan el éxito del cuerpo docente de una escuela en el desempeño de los estudiantes en pruebas estandarizadas. En Nicaragua, los únicos resultados que la gente asume como verdadero para medir el nivel de conocimiento tanto de los docentes como estudiantes de la secundaria, son los exámenes de admisión que se administran en las universidades públicas del país (Bermúdez, 2013).

Aunque no es el factor más importante en la evaluación de una escuela, de un docente o un estudiante, tenemos que comprender por qué una prueba estandarizada proporciona una evaluación equívoca sobre la eficacia del cuerpo docente de un colegio, simplemente por el hecho que no es aplicada a todo el estudiantado de secundaria. Ahora existe un estereotipo, que los estudiantes de ahora no saben o saben menos que los de antes, porque los docentes tampoco saben o saben menos que la escuela de antes.

También es común, que los docentes de la universidad digamos que el nivel de conocimiento en matemática de los estudiantes que ingresan del Sistema Educativo Medio al Superior es bajo, debido a su capacidad de razonamiento, redacción y sobre todo cuando les toca mostrar las habilidades para desarrollar un ejercicio de matemática que fue enseñado en la secundaria. Muchos de estos estudiantes no son capaces de visualizar operaciones matemáticas elementales, asimismo desconocen el significado de la misma. Esta situación se corrobora en los resultados del diagnóstico aplicado a sus profesores (Godino, 2002).

Ante esta situación no podemos permanecer indiferentes, como responsables de la formación de profesionales del país la universidad debe establecer un mecanismo que permita mejorar las capacidades tanto de estudiantes como de los docentes. Por esta razón, el presente documento presenta un diagnóstico específico en los docentes de secundaria del municipio de Juigalpa, el cual tiene como fin, establecer un mecanismo de cooperación entre el Ministerio de Educación (MINED) y la Facultad 
Regional Multidisciplinaria (FAREM) de Chontales, que permitirá mejorar la Educación Matemática en el municipio.

La investigación ha permitido establecer los factores que limitan la calidad de la formación de los nuevos estudiantes de secundaria, esto coadyuvará en el mejoramiento de los procesos de enseñanza de la matemática por parte de los profesores. Del mismo modo establece un vínculo universidad-comunidad, por medio de la implementación de talleres de capacitación en temas específicos de las áreas de matemática que se enseñan en la secundaria (Linares y Sánchez, 1990).

Específicamente brindará alternativas de solución a aquellas dificultades encontradas en el área de matemática tales como la lógica, aritmética, algebra, estadística. En donde directamente la universidad podrá aplicar una política de intervención en conjunto con el claustro de profesores de secundaria.

\section{Revisión de la literatura}

Durante muchos años como docente de secundaria, y en el transcurso de nuestro trabajo hemos observado diversos errores que cometen algunos colegas docentes de matemática. Por eso siempre hemos planteado una hipótesis: "Si el docente enseña correctamente hay más posibilidades que el alumno aprenda correctamente." ¿A qué se referirá esto?

Muchos docentes se esfuerzan por hacer que sus clases sean muy agradables y le imparten mucho planeamiento didáctico y muchas actividades que refuerzan el acto pedagógico del proceso de enseñanza-aprendizaje; pero muchas veces se olvidan de dedicarle más tiempo al aspecto cognitivo (académico) de la Educación Matemática, es decir, por bonitas y organizadas que estén las actividades de una hora de clase, estás no tendrán ningún sentido si el aspecto cognitivo no se desarrolla correctamente (INCE, 1998).

Hace muchos años una maestra enseñaba el cambio de escalas de Celsius a Fahrenheit utilizando la fórmula incorrectamente, su clase era muy alegre, después muchos estudiantes tenían grandes confusiones al hacer el proceso a la inversa y al pedir ayuda a otros colegas maestros de la misma asignatura, porque las respuestas no coincidían entre los docentes.

También un docente enseñaba que en la factorización de la forma ax $+b x+c$ los estudiantes sólo debían de buscar los factores que multiplicándolos dieran como resultado el valor de c, no importando el valor central b. Ella factorizaba así: x2 - 5x $-24=(x-6)(x+4)$. Cosa que cualquiera puede a simple vista notar que hay un grave error en la respuesta, porque debería ser $(x-8)(x+3)$. 
Mencionaremos una que nos llama mucho la atención por su generalización y porque algunos estudiantes usan este error con mucha frecuencia. Es el caso de la suma multiplicación y división de fracciones aritméticas, lo haré mediante un ejemplo que observé muchas veces en algunos salones de clases (¡Incluso en las secciones del 5to año, ahora undécimo grado!).

$$
x=\frac{\left(\frac{5}{3}\right) * 5+(-3)}{1+\left(\frac{5}{3}\right)}=2
$$

Lo que el profesor hizo fue cancelar el 5/3 del numerador y denominador y restó el 5 del 3 lo que dio como 2 y al dividirlo por 1 da como resultado 2, que técnicamente está correcta, pero el procedimiento empleado está totalmente incorrecto.

$$
x=\frac{\left(\frac{5}{3}\right) * 5+(-3)}{1+\left(\frac{5}{3}\right)}=2
$$

Este el procedimiento empleado por el profesor.

Es claro que un docente que permite esto a estudiantes del último grado, afecta no sólo el proceso de enseñanza aprendizaje sino que lo limita de sus capacidades cuando tenga que encontrarse con problemas de matemática parecidos, y los estudiantes estarán en seria desventaja frente a un examen de admisión que les dará la ubicación o no en una de las carreras universitarias (Backhoff, Tirado y Larrazolo, 2001).

El MINED plantea: Los docentes son actores claves del proceso educativo. La calidad de su formación, actualización y capacitación continua impacta decisivamente en la calidad de los aprendizajes de los educandos y su actuación pedagógica marca importantes pautas en el Desarrollo Humano de la población estudiantil (MINED, 2010).

Finalmente, como un mecanismo de apoyo pedagógico y fortalecimiento de la profesión docente, se implementan los Talleres de Evaluación, Programación y Capacitación Educativa (TEPCE). Estos son espacios de reunión mensual de los docentes de cada Núcleo Educativo para la programación y evaluación curricular. 
Los TEPCE han alcanzado un impulso importante, con diferentes funciones y alcances según cada Núcleo Educativo. Como un acompañamiento a la labor de los docentes, se ha impulsado también la Red de Acompañamiento Técnico Pedagógico (REDCAP), como una instancia vital para la mejora, desarrollo y adecuación del nuevo Currículo de la Educación Básica y Media. Su principal objetivo es apoyar y acompañar a los docentes en la aplicación de contenidos y generar intercambios entre diferentes niveles y ámbitos para la retroalimentación, evaluación, adecuación y adaptación curricular.

En la actualidad posee aproximadamente 7,364 miembros, docentes y especialistas de los niveles nacional, departamental, municipal y de núcleo. Los TEPCE en cuanto a espacio de intercambio colaborativo representan una potencial oportunidad para insertar acciones de actualización, formación, conocer y replicar mejores experiencias en marcha (MINED, 2010).

\section{Materiales y métodos}

La presente investigación es del tipo descriptivo-transversal y aunque se trabajó con una metodología cualitativa-cuantitativa, en este informe sobresalen los aspectos cuantitativos. La prueba se realizó durante el segundo TEPCE en el municipio de Juigalpa. Los participantes todo son profesores de matemáticas de secundaria, el día de la aplicación se encontraban veinte profesores, quienes después de hacérsele una introducción sobre los objetivos de la investigación aceptaron con beneplácito someterse a la prueba de evaluación que habían aplicado muchos de sus estudiantes en la UNAN-Managua, para el examen de admisión correspondiente al año 2014.

Se decidió realizarlo de una manera diferente al examen de admisión que se aplica en la UNAN-FAREM CHONTALES, en lugar de ser de opción múltiple se redactó la pregunta de tal modo que el docente tuviese que mostrar los procedimientos para encontrar la respuesta a cada ejercicio sugerido (Morales, 2012).

El análisis de los ítems, la discriminación, la confiabilidad validez de los mismos fue realizado con la metodología planteada por Kuder y Richardson (1937), quienes ofrecen estadísticos de los ítems basados en escalas dicotómicas y ordinales.

\section{Resultados y discusión}

Cuadro No. 1. Distribución de las respuestas del examen

\begin{tabular}{|l|r|r|r|}
\hline & Correcta & Incorrecta & \multicolumn{1}{|c|}{ NS/NR } \\
\hline E1 Respuesta del Ejercicio 1 Conjuntos & 5 & 10 & 5 \\
\hline E2 Respuesta del Ejercicio 2 Conjuntos & 1 & 18 & 1 \\
\hline
\end{tabular}




\begin{tabular}{|c|c|c|c|}
\hline & Correcta & Incorrecta & NS/NR \\
\hline E3 Respuesta del Ejercicio 1 Aritmética & 5 & 15 & 0 \\
\hline E4 Respuesta del Ejercicio 2 Aritmética & 6 & 10 & 4 \\
\hline E5 Respuesta del Ejercicio 1 Algebra & 12 & 2 & 6 \\
\hline E6 Respuesta del Ejercicio 2 Algebra & 2 & 13 & 5 \\
\hline E7 Respuesta del Ejercicio 3 Algebra & 7 & 10 & 3 \\
\hline E8 Respuesta del Ejercicio 4 Algebra & 3 & 14 & 3 \\
\hline Eg Respuesta del Ejercicio 1 Funciones & 2 & 8 & 10 \\
\hline E10 Respuesta del Ejercicio 2 Funciones & 4 & 5 & 11 \\
\hline E11 Respuesta del Ejercicio 1 Sucesiones & 6 & 7 & 7 \\
\hline E12 Respuesta del Ejercicio 2 Sucesiones & 6 & 6 & 8 \\
\hline E13 Respuesta del Ejercicio 1 Logaritmos & 2 & 10 & 8 \\
\hline E14 Respuesta del Ejercicio 2 Logaritmos & 1 & 8 & 11 \\
\hline E15 Respuesta del Ejercicio 1 G. Euclidiana & $\mathrm{O}$ & 17 & 3 \\
\hline E16 Respuesta del Ejercicio 2 G. Euclidiana & 0 & 12 & 8 \\
\hline E17 Respuesta del Ejercicio 3 G. Euclidiana & 9 & 10 & 1 \\
\hline E18 Respuesta del Ejercicio 1 Trigonometría & 1 & 12 & 7 \\
\hline E19 Respuesta del Ejercicio 2 Trigonometría & o & 9 & 11 \\
\hline E2o Respuesta del Ejercicio 3 Trigonometría & 6 & 4 & 10 \\
\hline E21 Respuesta del Ejercicio 1 G. Analítica & 6 & 6 & 8 \\
\hline E22 Respuesta del Ejercicio 2 G. Analítica & 5 & 5 & 10 \\
\hline E23 Respuesta del Ejercicio 1 Estad y Probab & 2 & 15 & 3 \\
\hline E24 Respuesta del Ejercicio 2 Estad y Probab & 3 & 17 & $\mathrm{O}$ \\
\hline E25 Respuesta del Ejercicio 3 Estad y Probab & 6 & 14 & $\mathrm{O}$ \\
\hline
\end{tabular}

Número de respuestas e incorrectas por área matemática del examen aplicado

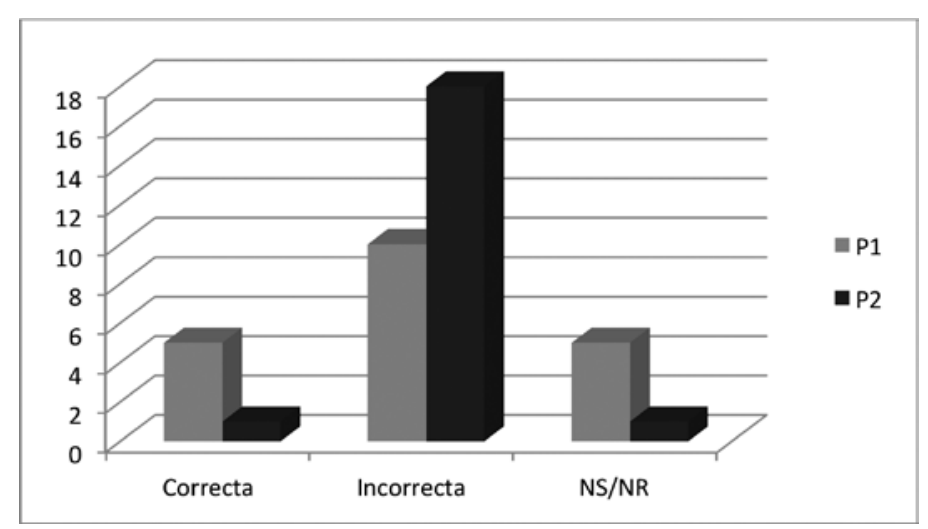

Figura No.1. Lógica y conjuntos 
La figura No. 1 refleja el número de maestros que respondieron de forma correcta e incorrecta las primeras dos preguntas que corresponden al área de lógica y teoría de conjuntos, se puede notar que del total que respondieron la pregunta no 1,referida al conjunto potencia de un conjunto fue la que más respuestas correctas obtuvo con un total del $30 \%$ de los que la respondieron; pero concerniente a la habilidad para representar Diagramas de Venn-Euler se obtuvo un bajo porcentaje de respuestas correctas, únicamente el $5 \%$ (un docente), siendo está temática una de los contenidos básicos que se imparte en el séptimo grado, pero hay dificultades en los docentes para representar más de dos conjuntos con operaciones indicadas.

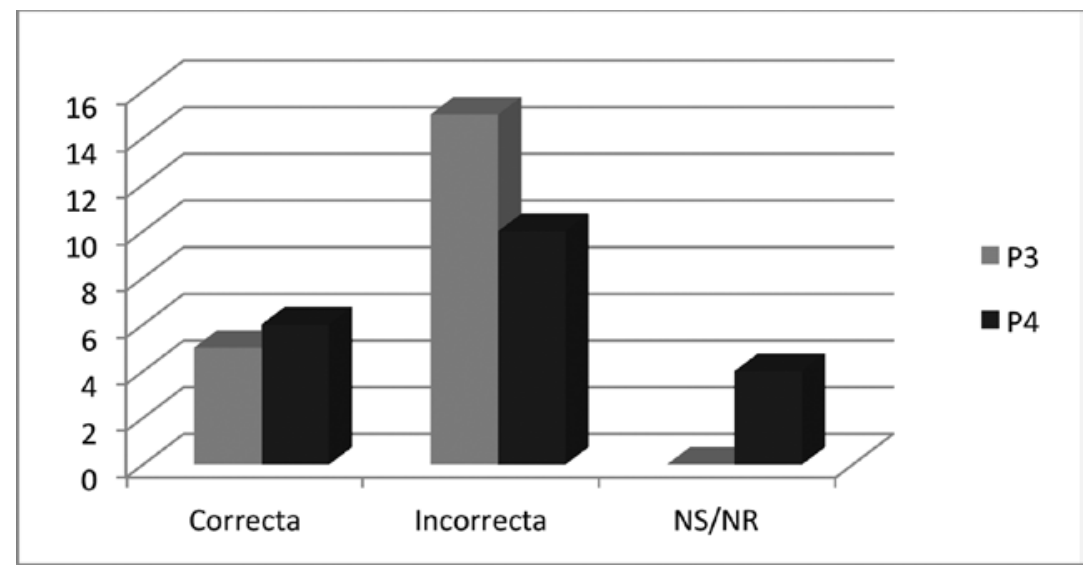

Figura No. 2. Aritmética

Cuando se analiza el comportamiento de respuestas correctas e incorrectas en el área de aritmética, la figura No. 2 refleja que estos porcentajes son muy bajos en comparación a lo esperado; puesto que son ejercicios referidos a operaciones básicas que se enseñan desde la primaria, pero surge una dificultad cuando hay que usar estas operaciones en problemas sencillos de reparto, ya que menos del $40 \%$ de los docentes pudieron responder correctamente alguna de las dos preguntas.

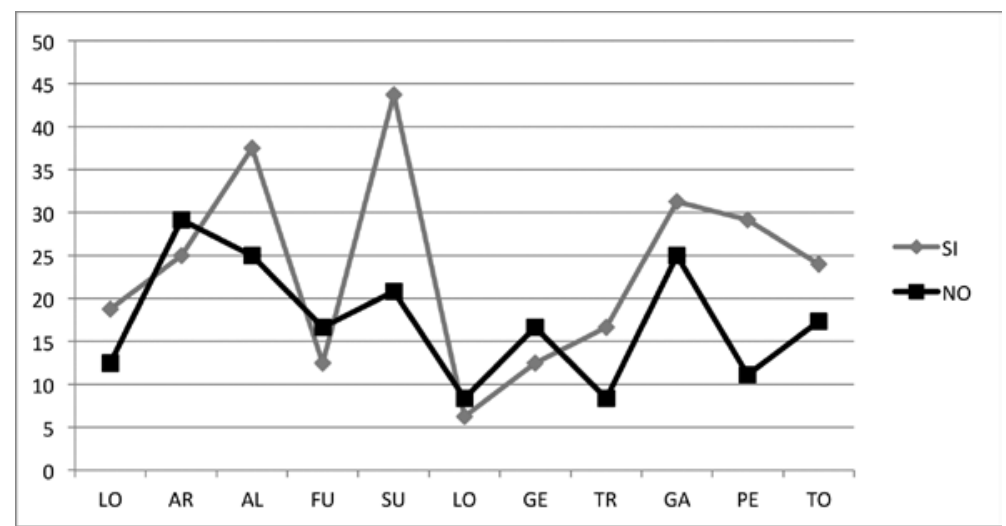

Figura No. 3. Promedio obtenido vs si trabaja o no en $10 \mathrm{mo}$ y 11mo grado 
Tratando de establecer si existen factores que diferencien a un grupo de maestros de otro grupo, en la figura No. 3 se trazó el promedio obtenido por los docentes en cada área del conocimiento de acuerdo a si trabajan o no en los décimos y undécimos grados, pensando en quienes trabajan en estos grados son los que mejor están preparados; el resultado indica que en algunas áreas si se correspondió así, pero en lógica, algebra, funciones, logaritmos, geometría, los promedios fueron estadísticamente iguales. Aunque es destacable que estos maestros de los grados superiores, las calificaciones a excepción de algebra y geometría euclidiana, fueron mayores a los que no imparten estos grados. Por tanto debe pensarse en una rotación del personal como alternativa para combatir el estancamiento.

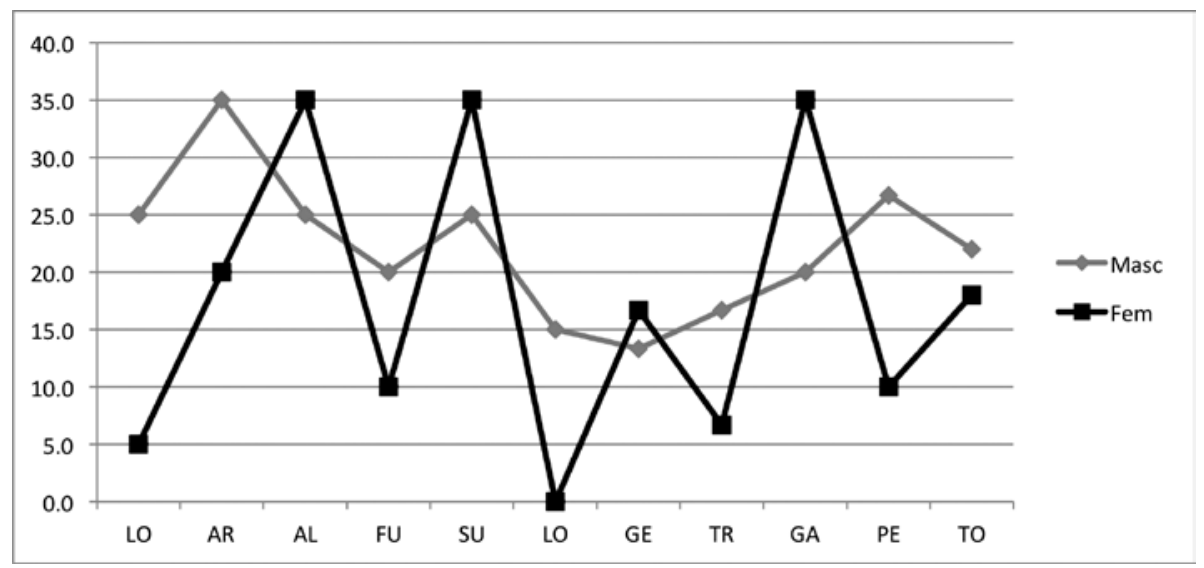

Figura No. 4. Promedio obtenido vs género del docente

Del mismo modo, la figura No. 4 presenta los promedios obtenidos en las diferentes áreas evaluadas versus el género del docente, con la idea de determinar si hay un sector (femenino o masculino) que responde mejor este examen, pero esta variable solo mostró que el género masculino fue más consistente en las respuestas que brindó, porque el género femenino obtuvo notas muy altas pero también muy bajas, inclusive cero, las áreas del conocimiento se reparten en proporciones casi iguales; por lo tanto, no podemos argumentar que el género determina si un docente está mejor preparado o no para resolver este examen.

\section{Conclusiones}

Se puede afirmar que dentro de las competencias cognitivas del profesorado que imparte secundaria en la ciudad de Juigalpa estas son muy limitadas y no cuentan con suficientes argumentos como para asegurar una educación científica de calidad, misma que promueve el MINED. 


\section{EDUCACIÓN}

Dentro de las limitantes, tenemos el escaso conocimiento a nivel medio de las competencias elementales para que un estudiante pueda rendir óptimamente en un examen de admisión de alguna universidad pública, y casi todas las áreas del conocimiento están en un mismo estado, lo que hace que este sector requiera intervención por parte de una instancia superior para mejorar el nivel mostrado hasta ahora.

Las áreas en las que mejor se desempeñaron los maestros en la prueba fueron en: El cálculo de las raíces de una ecuación cuadrática. Cálculo de términos y suma de sucesiones aritméticas. Propiedades de los lados y ángulos de un triángulo. Comprobación de identidades básicas. Conocimiento básico sobre las propiedades de las ecuaciones paralelas y perpendiculares, así como conocimiento elemental de los elementos de una elipse y su ecuación.

Aunque en lo general, no existe ningún área específica en la que los docentes se hayan mostrado fuertes, lo mencionado en el párrafo anterior obedece sólo a los ítems donde estos docentes superan el 6o\% de respuestas correctas.

Se contrastó descriptivamente que no existen factores determinantes para la obtención de puntajes altos en la prueba, se pudo obtener que aquellos docentes que impartían el décimo y undécimo grado pudieran salir mejor en algunas áreas del conocimiento, por tanto no podemos generalizar este resultado. Podemos decir que tampoco el género influyó en los resultados obtenidos y que el problemas debería resolverse contrastando otras variables diferentes a las expuestas acá.

En este apartado del análisis pudo haberse usado las pruebas de hipótesis de la estadística no paramétrica, pero, aunque se hicieron en la búsqueda de resultados, no se incluyeron porque carecían de significado estadístico tantas pruebas realizadas a cada área del conocimiento cuando obtenemos los mismos resultados con las figuras mostradas anteriormente.

Se debe realizar una reunión con las autoridades del MINED y UNAN-FAREM CHONTALES con el fin de conocer estos resultados del diagnóstico y formular una estrategia a corto y mediano plazo para el fortalecimiento de la Educación Matemática en la secundaria, comenzando en el municipio de Juigalpa para después poder ampliarlo a todo el departamento de Chontales.

Asimismo, la estrategia deberá contener acciones de la universidad hacia el MINED y este deberá abrir los espacios para que los estudiantes de la universidad se involucren desde las prácticas de familiarización. En primera instancia se pueden aprovechar los TEPCES para brindar capacitaciones en los temas específicos de matemática hasta poder ampliar el proyecto a un estudio más sistemático y permanente. 


\section{Recomendaciones}

- Iniciar el proceso de capacitación a lo inmediato, ya que se encontraron muchas dificultades en todas las áreas del conocimiento matemático.

- En cada dos horas del TEPCE, desarrollar un taller de matemática donde se aborden temáticas comunes al examen de admisión y lo que se enseña en la secundaria. Este taller debe ser promovido y orientado por el MINED, en donde la universidad y/o MINED, faciliten los docentes mejor preparados en cada área del conocimiento.

- El MINED debe elaborar un proyecto en conjunto con la universidad a corto plazo para fortalecer la asignatura de matemática en este 2015. En el cual debe involucrar a todos los docentes del municipio y luego promover la ampliación del mismo al departamento.

- Revisar las competencias académicas de los docentes que imparten docencia en los grados de décimo y undécimo, por medio de un diagnóstico específico, mediante observaciones, entrevistas y pruebas de control de la calidad.

- Implementar un programa de selección de docentes para el 2015 - 2016 de acuerdo con las competencias que muestren en una prueba de matemática, para que estos sean los que trabajen en los grados superiores y además puedan apoyar a sus colegas maestros de los grados más bajos.

- Establecer una metodología de rotación de docentes por los diferentes grados que se imparten para que los mismos no se estanquen académicamente. Esto puede hacerse año con año, en dependencia de la evolución de los conocimientos en las pruebas de control que el MINED pueda establecer.

- El MINED Juigalpa debe establecer un mecanismo para asegurarse que el docente que imparte un grado determinado tiene el dominio académico suficiente para impartir clases de matemática; esto lo puede hacer con un examen al inicio e intermedio de cada año, que evalúe las competencias de los docentes, en función de mejorar la calidad académica. 


\section{Lista de referencias}

Backhoff, E; Tirado, F y Larrazolo, N; (2001). Ponderación diferencial de reactivos para mejorar la validez de una prueba. Universidad Autónoma de Baja California. Revista electrónica de investigación educativa. Ensenada México. Disponible en: http://redie.uabc.mx/contenido/vol3no1/contenido-tirado.pdf

Bermúdez, J. C. (21 de Enero de 2013). Maestros aplazados. La Prensa. Disponible en http://www.laprensa.com.ni/2013/o1/21/ reportajes-especiales/131574-maestros-aplazados

Godino, J.D. (2002). Competencia y comprensión matemática: ¿qué son y cómo se consiguen?. UNO. Revista de Didáctica de la Matemática, 29, 9-19.

INCE (1998). Diagnóstico General del Sistema Educativo. Avance de Resultados. MEC, Madrid.

Kuder, G. F., y Richardson, M. W. (1937). The theory of the estimation of test reliability. Psychometrika, 2(3), 151-160.

Linares, S. y Sánchez, V. (1990). El conocimiento profesional del profesor de matemáticas y la enseñanza de las matemáticas. En Linares, S. y Sánchez, V (eds.), Teoría y práctica en Educación Matemática, Alfar, Sevilla.

Ministerio de Educación (2010). Plan estratégico 2011 - 2015, Managua, Nicaragua.

Morales, P; (2012). Análisis de items en las pruebas objetivas. Universidad Pontificia Comillas, pág. 1-16. 